\title{
Helina
}

\section{Activities of the Pan African Health Informatics Association}

\section{Prepared by Ghislain B. Kovematchoua Tchuitcheu}

\section{HELINA 2018}

The HELINA Board put out a Call for Bids to host HELINA 2018 in June 2017. The Kenya Health Informatics Association presented a bid and was given approval to host the conference in Nairobi, Kenya from 1 st -5 th October 2018. It is expected that the region will build on successes of past conferences and will make HELINA 2018 a formidable event.

The conference will focus on how technology is being used to strengthen health systems in the African Region. Issues of specific interest are the development and implementation of integrated e-Health plans and policies that enable capacity building for eHealth professionals, improved quality of health information, and promotion of the meaningful use of health data to support and ground decision-making. Also in focus will be enabling access to essential medical supplies through improved supply chain and logistics, development of sustainable health information systems for service delivery, and innovative health financing models that improve access to health. There is also an interest in the role of digital health in health surveillance systems particularly due to emerging health threats from non-communicable diseases (NCDs) and the core participatory role of the client in detection, response, treatment, and care. Special attention will be paid to the role of e-Health in achieving the Sustainable Development Goals (SDG) voted by the UN in September 2015 and specifically to goal 9, target 9c which aims to "Significantly increase access to information and communications technology and strive to provide universal and affordable access to the Internet in the least developed countries by 2020 “.

\section{HELINA Conference 2017}

The Conference attracted 198 participants from 21 nationalities. The pre-conference workshops had 83 participants and was appreciated by attendants similarly to the workshop in Accra, Ghana in 2015. HELINA plans to maintain this conference model. While participant feedback was positive and quite enthusiastic, there was unfortunately weak political backing for the conference, evident in the very few representatives from the Ministry of Health. The conference papers were published in a special issue of the Journal of Health Informatics in Africa (JHIA) - www.jhia-online.org.

\section{HELINA Education Working Group}

The Education WG held a successful meeting at the HELINA 2017 conference "Integrated Health Information Management Resources for Global Healthcare Strategies" in Bujumbura, Burundi, April 2017.

One of the WG members Aurore Nishimwe presented a paper "Assessment of Health Informatics Competencies in Undergraduate Training of Healthcare Professionals in Rwanda" using the IMIA Recommendations on Education as the tool for her survey. Her survey demonstrated that the use of the recommendations was perceived as highly appropriate. Dr. Frank Verbeke and Profs Marc Nyssen and Graham Wright had also used the IMIA Education Recommendations together with the IMIA Knowledge Base to evaluate a postgraduate program in Rwanda with success.

Earlier this year the IMIA Board asked President-Elect Sabine Koch to oversee and update the current IMIA Recommendations on Education. Co-authors of the existing document as well as younger educators have been invited to participate including the Education WG Chair. This effort was presented at the Education WG meeting and Aurore offered to email the members present $(40+)$ a copy of the IMIA paper and her work. It was agreed that the Chair would undertake a literature review of the use of the IMIA Education Recommendations in Africa and once completed the results sent to members.
A request has been made to the recipients to comment on the appropriateness of the recommendations for Africa so that the Chair can forward a response to the IMIA review team. Apparently, the IMIA Recommendations on Education have an important role to play in Africa, which is only now catching up with the formalisation of Health Informatics education. The HELINA Education WG has an opportunity to influence the review.

HELINA Education WG website http:// wg-education.helina-online.org/

LinkedIn Group https://www.linkedin.com/ groups $/ 8274552$

Youtube repository HI Videos https://www. youtube.com/channel/UCXagzt4NPZvj9FYYtbHc5vQ

\section{HELINA Data Mining and Big Data Analytics Working Group}

Within the preconference meetings and workshops, on Sunday 23rd April 2017, a tutorial was organized by the WG and lectured by the Chair, Dr. Georges Nguefack-Tsague on "Introduction to data mining and big data analytics methods and techniques". The tutorial was followed by questions, answers, and discussions. There were 50 participants from Benin, Burundi, Cameroon, DRC, Ghana, Guinea, Kenya, Rwanda, and Tanzania. Participants were very enthusiastic about data mining and appreciated the course content. Following the tutorial, Dr. Georges Nguefack-Tsague also conducted a hands-on training session on "Putting data warehousing and data mining into practice". This practical session focused on "Application of data mining methods on real world African health data sets". The training followed the main recommendation of participants of the workshop on "Data Mining and Big Data Analytics" held in Accra, Ghana on March 7, 2015. Data for the practical training contained 20 million medical records from patients from West and Central Africa: Burundi, Rwanda, Tanzania, Democratic Republic of 
Congo (DRC), and Mali. These data were collected at the point of care with the open source integrated hospital information management system OpenClinic GA. The $\mathrm{R}$ package Rattle software was used during that session. The session included Problem Understanding, Data Understanding, Data Preparation, Modeling, Evaluation, and Deployment. The building models included Predictive Analytics, Cluster Analysis, Association Analysis, Decision Trees, Random Forests, Boosting, and Support Vector Machines.

A meeting of the WG was organized on Tuesday, 25th April where 25 participants from five nations (Belgium, Burundi, DRC, Guinea, and Rwanda) enrolled as members of the WG.

\section{HELINA Nursing Special Interest Group}

The Nursing SIG has started to raise interest in Nursing Informatics within Africa and wishes to thank the support of the HELINA Board and in particular Dr. Ghislain Kouematchoua, its Chairman.

The group had a high presence at the HELINA 2017 conference "Integrated Health Information Management Resources for Global HealthCare Strategies" in Bujumbura, Burundi, April 2017. The NSIG Chair, Prof. Graham Wright presented the Keynote address "Mobile ICT-tools for nurses in rural clinics" at the opening of the conference.

Dr. Ghislain Kouematchoua introduced the members of the HELINA Nursing SIG panel to a full audience of more than 200 . He reiterated the Board's commitment to include Nursing in all future HELINA conferences and gave a brief overview of the importance of training Nurses in Informatics within Africa. Dr. Evelyn Hovenga, a Nursing Informatics Professor from Australia, presented an overview of Nursing Informatics as a discipline and its place in Health. The video is available at https://www.youtube. $\mathrm{com} /$ watch? $\mathrm{v}=$ qyZp4SKaTwk

The panel consisting of Dr. Frank Verbeke, Dr. Helen Wright and Prof. Graham Wright spent over an hour answering questions in English and French to an enthusiastic audience. One of the main outcomes was a motion that the International Classification for Nursing Practice (ICNP) system be adopted in Africa.
The International Classification for Nursing Practice (ICNP), a product of the International Council of Nurses (ICN), is a formal terminology endorsed by WHO. It provides a dictionary of terms and an expressive relationship that nurses can use to describe and report their practice in a systematic way. The resulting information is used reliably to support care and effective decision-making and inform nursing education, research, and health policy. ICNP is intended for use by and for nurses. It is a rich and comprehensive resource that nurses can use to describe and report in detail the things that they assess (diagnoses e.g. nausea) and the things that they provide (interventions e.g. counselling).

Dr. Frank Verbeke confirmed that the ICNP would be introduced to all the OpenClinic GA systems in Africa, a major initiative, which was supported with enthusiasm.

\section{News from the National Societies}

\section{Cameroon}

The Cameroonian Medical Informatics Association organised the Sixth Cameroonian day of Medical Informatics from 14th - 15th November, 2017, in Yaoundé, Cameroon. The main topic of the conference was "Social network and medical practice in Cameroon".Journal of Health Informatics in Africa (JHIA)

\section{Kenya}

The Kenya Health Informatics Association (KeHIA) engaged in the following events:

1. MEASURE Evaluation, in conjunction with the Kenya Health Informatics Association, hosted a one-day workshop on Wednesday, 7th May, 2017 in Nairobi, Kenya. This workshop was organised to review a HIS interoperability maturity model and a companion assessment tool. Input provided by experts at this forum has resulted in a tool that is freely available to organisations and countries looking at HIS Interoperability issues. The association also co-hosted another workshop with MEASURE Evaluation on Thursday, 8th June, 2017. The purpose of this workshop was to review guidelines for mHealth data privacy, confidentiality, and security. This has resulted in guidelines that are freely available to countries and organisations engaged in mHealth projects.

2. The Kenya Health Informatics Association hosted a one-day symposium at the Strathmore University, Nairobi, Kenya on Friday, 28th April 2017. This symposium was the second in a series of stand-alone "informatics hot spot" symposiums aimed at providing an informatics "market place" for learning, sharing, and networking. There were both formal presentation slots and moderated "fishbowl" sessions for sharing and learning. There was a rich and diverse audience comprising of representatives from both the public and private health sector, national and county government, academia, CSOs, and tech hubs. The goal of this second series of an "Informatics Day" was to provide a well-balanced conferenced and un-conferenced opportunity to explore current activities in Kenya concerning informatics investments, interventions, and impacts, to meet the real people behind those digital health investments, interventions, and impacts, and to connect and establish meaningful, mutually beneficial informatics investment and intervention relationships. This meeting was a partnership between the Ministry of Health (MOH) (Division of Monitoring and Evaluation, Health Research Development and Informatics), KEMRI-Wellcome Trust, and Strathmore University School of Business. The inaugural series focused on "'Big Data and Analytics".

\section{Acknowledgements}

The author would like to thank the WG chairs Prof. Graham Wright and Dr. Georges Nguefack-Tsague, and HELINA secretary Frances da-Costa Vroom for their contribution to this report.

\section{Regional Editor}

Ghislain B. Kouematchoua Tchuitcheu, $\mathrm{PhD}$, MIAHSI

IMIA Vice President for HELINA

E-mail: kouematchoua@helina-online.org ghislain.k@koegni-ehealth.org

www.helina-online.org 